Doklady Mathematics, 2012, Vol. 86, No. 1, pp. 587-590 (doi:10.1134/S1064562412040035)

\title{
Stability domain analysis of an antiwindup control system for an unstable object
}

Leonov G.A., Kuznetsov N.V., Pogromskii Yu.

Draft $^{1}$

\begin{abstract}
At low speeds, modern fighters become unstable. For their stabilization in fighter's control system is used anti-windup scheme. This report is devoted to mathematical model of anti-windup and its non-linear analysis. For investigation of existence of oscillations the method of comparison systems, Andronov's point-transformation method and the estimation of trajectories on Poincare cross-section are used. These methods allow us to localize unstable limit cycle and to estimate the region of stability of the system. Simulation of the system shows the accuracy of the analytical estimates.
\end{abstract}

Keywords: aircraft, windup, antiwindup, pilot induced oscillation, hidden oscillation, nonlinear control system, saturation, absolute stability

\section{INTRODUCTION}

In the 50-60s of the last century the investigations of widely known Markus-Yamabe's, Aizerman's, and Kalman's conjectures on absolute stability have led to the finding of hidden oscillations (a basin of attraction of which does not contain neighborhoods of equilibria) in automatic control systems with a unique stable stationary point and with a nonlinearity, which belongs to the sector of linear stability (see, e.g., $[3,4,1,6$, $2,7])$.

At the end of the last century the difficulties of numerical analysis of hidden oscillations arose $[8\rfloor$ in simulation of aircraft's control systems (anti-windup scheme) and caused aircraft crashes: "Since stability in simulations does not imply stability of the physical control system (an example is the crash of the YF22 [Boeing]), stronger theoretical understanding is required," since standard computational procedure (in which after a transient process a trajectory, started from a point of unstable manifold in a neighborhood of equilibrium, reaches a state of oscillation therefor one can easily identify it) can not reveal such hidden oscillations.

Nowadays, it is believed [9] that the crashes of a US Boeing YF-22 Raptor in April, 1992 [10] and a Swedish Saab Gripen [11] were caused by the synthesis of control algorithms without saturation-type nonlinearities taken into account. In these cases, a pitch flutter (i.e., pitch oscillations with an increasing amplitude) was observed while the plane was landing. This effect is known as windup, while windup-eliminating correcting schemes and devices are known as anti-windup $[17,13,14,16,15,12$. Recall that, in the 1940s, Keldysh [18] developed mathematical methods for analyzing various flutter-suppressing systems for planes. In this paper, Keldysh's methods are developed and modified in order to study anti-windup.

\section{STATEMENT OF THE PROBLEM}

Consider the following flowchart of an anti-windup control system [17] for a first-order unstable object.

The flowchart presented in Fig. 1 is equivalent to the dynamical system

$$
\begin{aligned}
& \dot{x}=a x-\operatorname{sat}(\sigma) \\
& \dot{\sigma}=(k a+b) x-b m \sigma+(b m-k) \operatorname{sat}(\sigma)
\end{aligned} \quad \operatorname{sat}(\sigma)= \begin{cases}\operatorname{sign}(\sigma) & |\sigma| \geqslant 1 \\
\sigma & |\sigma|<1\end{cases}
$$

\footnotetext{
${ }^{1}$ Nikolay V. Kuznetsov, nkuznetsov239 at gmail.com (correspondence author)
} 


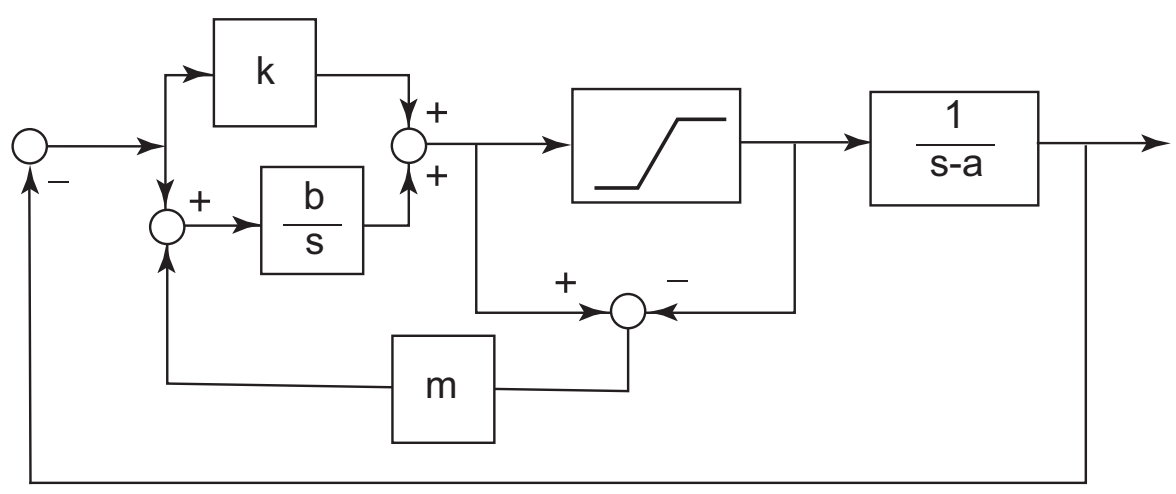

Figure 1: Flowchart of an antiwindup control system.

where $a, b, m$, and $k$ are positive parameters. The matrix of the linear part of system (2.1) is unstable, and its eigenvalues are $a$ and $-b m$, while the matrix of the system obtained taking into account the linear segment of the nonlinearity $\operatorname{sat}(\sigma)$ with $|\sigma|<1$ has the eigenvalues

$$
\frac{-(k-a) \pm \sqrt{d}}{2}, \quad d=(k-a)^{2}-4 b .
$$

Assume that the system is stable in the absence of saturation; i.e.,

$$
k>a .
$$

System (2.1) has a stable equilibrium at the point $(0,0)$, and two additional symmetric saddle equilibria

$$
\sigma= \pm\left(1+\frac{1}{a m}\right), x= \pm \frac{1}{a} .
$$

appear when an anti-windup link is considered in a feedback loop (i.e., $m \neq 0$ ). The qualitative behavior of the trajectories of the system is shown in Fig. 2. Here, two separatrices tending to two symmetric saddle equilibria, respectively, are wound off the unstable limit cycle enclosing the stable zero equilibrium. The relative positions of the saddles and the limit cycle in Fig. 2 suggest that the stability domain expands and tends to infinity as the parameter $a$ monotonically approaches zero.

Let us estimate the domain of initial data for which the solutions of the system are bounded and tend to the zero equilibrium.

\section{ANALYSIS OF EXISTENCE OF A LIMIT CYCLE}

Following $[18,20,21\rfloor$, we use the point map method [19] to find the conditions for the existence of a limit cycle in system (2.1). Consider the following system on the linear segments of nonlinearity:

$$
\begin{array}{ll}
\operatorname{sat}(\sigma)=-1: & \left\{\begin{array}{l}
\dot{x}=a x+1 \\
\dot{\sigma}=(k a+b) x-b m \sigma-(b m-k)
\end{array}\right. \\
\operatorname{sat}(\sigma)=\sigma: & \left\{\begin{array}{l}
\dot{x}=a x-\sigma \\
\dot{\sigma}=(k a+b) x-k \sigma
\end{array}\right. \\
\operatorname{sat}(\sigma)=1: \quad\left\{\begin{array}{l}
\dot{x}=a x-1 \\
\dot{\sigma}=(k a+b) x-b m \sigma+(b m-k)
\end{array}\right.
\end{array}
$$




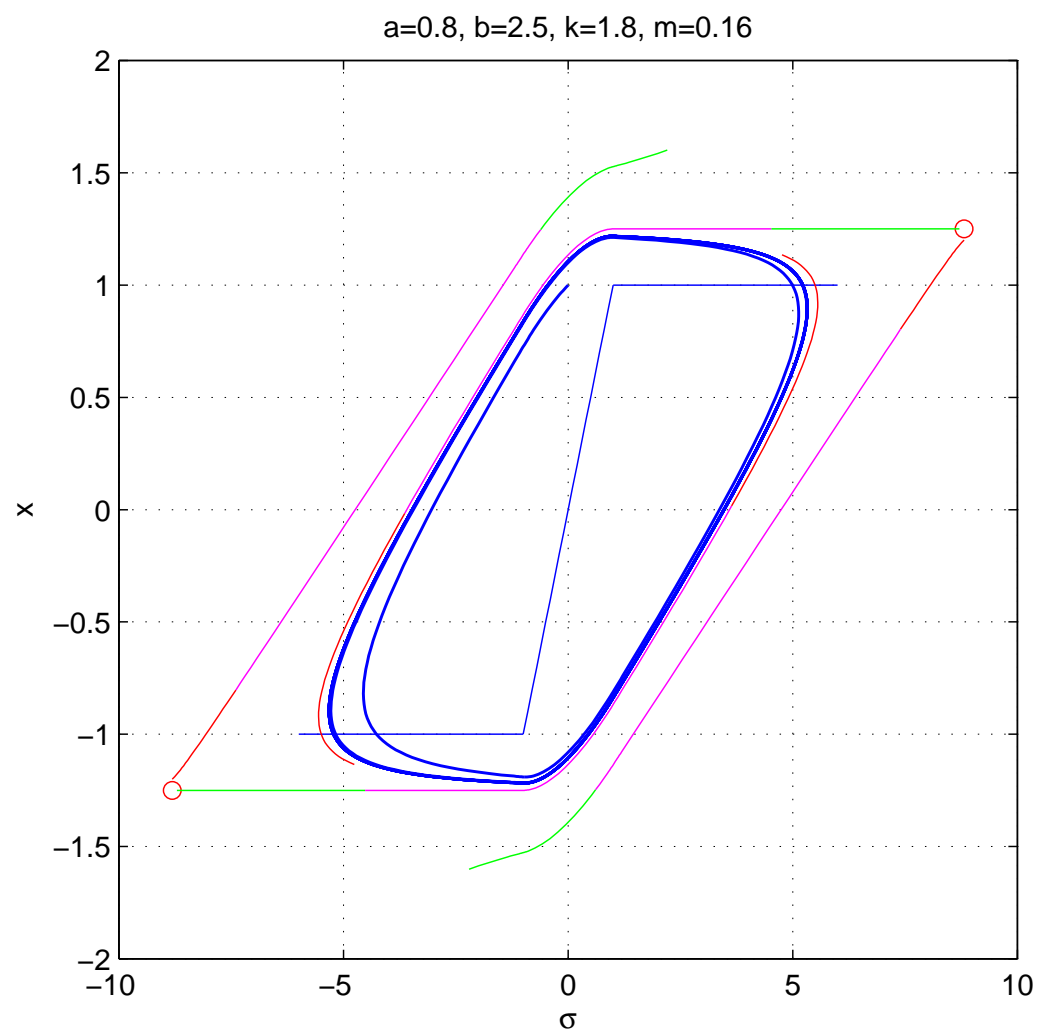

Figure 2: Qualitative behavior of the trajectories of the system $(\mathrm{a}=0.8, \mathrm{~b}=2.5, \mathrm{k}=1$, and $\mathrm{m}=0.16)$.

Note that, if $(x(t), \sigma(t))$, is a solution of the system, then $(-x(t),-\sigma(t))$ is a solution as well. Integrating 
the system over the linear segments of nonlinearity yields the solutions of the system

$$
\begin{aligned}
& \sigma \leq-1: \quad\left\{\begin{array}{c}
x_{1}\left(t, \sigma_{0}, x_{0}\right)=-\frac{1}{a}+\frac{e^{a t}}{a}\left(1+a x_{0}\right) \\
\sigma_{1}\left(t, \sigma_{0}, x_{0}\right)=\frac{-m(a k+b) x_{0}+m(a+b m) \sigma_{0}+m(b m+a-k)+1}{(b m+a) m} e^{-b m t}+ \\
+\frac{(k a+b)\left(1+a x_{0}\right)}{a(b m+a)} e^{a t}-\left(1+\frac{1}{a m}\right)
\end{array}\right. \\
& \left(x_{2}\left(t,-1, x_{0}\right)=\frac{1}{2 \sqrt{d}}\left((\sqrt{d}+(k+a)) x_{0}+2\right) e^{\frac{-(k-a)+\sqrt{d}}{2} t}+\right. \\
& |\sigma|<1, d>0:\left\{\begin{aligned}
& +\frac{1}{2 \sqrt{d}}\left((\sqrt{d}-(k+a)) x_{0}-2\right) e^{\frac{-(k-a)-\sqrt{d}}{2} t} \\
\sigma_{2}\left(t,-1, x_{0}\right)= & \frac{1}{2 \sqrt{d}}\left(2(k a+b) x_{0}+a+k-\sqrt{d}\right) e^{\frac{-(k-a)+\sqrt{d}}{2} t}-
\end{aligned}\right. \\
& -\frac{1}{2 \sqrt{d}}\left(2(k a+b) x_{0}+a+k+\sqrt{d}\right) e^{\frac{-(k-a)-\sqrt{d}}{2} t} \\
& |\sigma|<1, d=0:\left\{\begin{array}{l}
x_{2}\left(t,-1, x_{0}\right)=\frac{1}{2} e^{(-k+a) t / 2}\left(\left(x_{0}(k+a)+2\right) t+2 x_{0}\right) \\
\sigma_{2}\left(t,-1, x_{0}\right)=\frac{1}{4} e^{(-k+a) t / 2}\left(\left((k+a)^{2} x_{0}+2 k+2 a\right) t-4\right)
\end{array}\right. \\
& |\sigma|<1, d<0:\left\{\begin{array}{c}
x_{2}\left(t,-1, x_{0}\right)=\frac{1}{2 \sqrt{d}} e^{(-k+a) t / 2}\left(\sin (\sqrt{d} t)\left((k+a) x_{0}+2\right)+2 x_{0} \cos (\sqrt{d} t) \sqrt{d}\right) \\
\sigma_{2}\left(t,-1, x_{0}\right)=\frac{1}{4 \sqrt{d}} e^{(-k+a) t / 2}\left(\sin (\sqrt{d} t)\left(\left((k+a)^{2}+4 d\right) x_{0}+2 a+2 k\right)-\right. \\
-4 \cos (\sqrt{d} t) \sqrt{d})
\end{array}\right. \\
& \sigma \geq 1: \quad\left\{\begin{array}{c}
x_{3}\left(t, \sigma_{0}, x_{0}\right)=\frac{1}{a}+\frac{e^{a t}}{a}\left(-1+a x_{0}\right) \\
\sigma_{3}\left(t, \sigma_{0}, x_{0}\right)=\frac{-m(a k+b) x_{0}+m(a+b m) \sigma_{0}+m(k-b m-a)-1}{(b m+a) m} e^{-b m t}+ \\
+\frac{(k a+b)\left(-1+a x_{0}\right)}{a(b m+a)} e^{a t}+\left(1+\frac{1}{a m}\right)
\end{array}\right.
\end{aligned}
$$

By using formulas (3.4) and the system of equations

$$
\left\{\begin{array}{l}
x_{2}\left(t_{1},-1, x_{0}\right)=\widetilde{x} \\
\sigma_{2}\left(t_{1},-1, x_{0}\right)=1 \\
x_{3}\left(t_{2}, 1, \widetilde{x}\right)=-x_{0} \\
\sigma_{3}\left(t_{2}, 1, \widetilde{x}\right)=1
\end{array}\right.
$$

we determine the initial conditions $\sigma=-1, x=x_{0}>0$ on the limit cycle of the system.

The resulting system can be simplified using the method of comparison systems, which is described below. 


\section{CONSTRUCTION OF COMPARISON SYSTEMS FOR STA- BILITY DOMAIN ESTIMATION}

Following $\lfloor 20,21\rfloor$, the domain of attraction of the zero equilibrium of system (2.1) is analyzed by applying the method of comparison systems [21]. Define the function

$$
\operatorname{sign}_{+1}(\sigma)=\left\{\begin{array}{ll}
1, & \sigma>1 \\
-1, & \sigma<1
\end{array} \quad \operatorname{sign}_{-1}(\sigma)= \begin{cases}1, & \sigma>-1 \\
-1, & \sigma<-1\end{cases}\right.
$$

To estimate the basin of attraction of the zero equilibrium of the original system from below, we consider the comparison system

$$
\begin{aligned}
& \dot{x}=a x-\varphi_{1}(\sigma, x) \\
& \dot{\sigma}=(k a+b) x-b m \sigma+(b m-k) \varphi_{1}(\sigma, x)
\end{aligned} \quad \varphi_{1}(\sigma, x)= \begin{cases}\operatorname{sign}_{+1}(\sigma), & (1+a m) x-m \sigma>0 \\
\operatorname{sign}_{-1}(\sigma), & (1+a m) x-m \sigma<0\end{cases}
$$

Similarly, the comparison system

$$
\begin{aligned}
& \dot{x}=a x-\varphi_{2}(\sigma, x) \\
& \dot{\sigma}=(k a+b) x-b m \sigma+(b m-k) \varphi_{2}(\sigma, x)
\end{aligned} \quad \varphi_{2}(\sigma, x)= \begin{cases}\operatorname{sign}_{-1}(\sigma), & (1+a m) x-m \sigma>0 \\
\operatorname{sign}_{+1}(\sigma), & (1+a m) x-m \sigma<0\end{cases}
$$

is constructed to estimate the basin of attraction of the zero equilibrium from above.

Consider the conditions under which each comparison system has a limit cycle enclosing the zero equilibrium. In view of the nonlinearities chosen in (4.6) and (4.7), this cycle is the boundary of a positively invariant (or negatively invariant) set of system (2.1).

Figure 4 shows the construction of limit cycles in comparison systems for $a=0.8, b=2.5, k=5, m=0.05$.

In view of the symmetry, for comparison system (4.6) to have a periodic solution, we assume that there is a point $\sigma=-\sigma_{0}<-1, x=-h \sigma_{0}$ such that

$$
\left\{\begin{array}{l}
x\left(t_{1},-\sigma_{0},-h \sigma_{0}\right)=\widetilde{x} \\
\sigma\left(t_{1},-\sigma_{0},-h \sigma_{0}\right)=1 \\
x\left(t_{2}, 1, \widetilde{x}\right)=h \sigma_{0} \\
\sigma\left(t_{2}, 1, \widetilde{x}\right)=\sigma_{0}
\end{array}\right.
$$

for some positive values of $t_{1}, t_{2}$ and $\widetilde{x}$. Here, $h=\frac{m}{(1+a m)}$ and the considered solutions are determined by the corresponding formulas in (3.4). Finding $t_{1}$ and $t_{2}$ from the equations for $x$, namely,

$$
t_{1}=\frac{1}{a} \log \left(\frac{1-a \widetilde{x}}{1+a h \sigma_{0}}\right), t_{2}=\frac{1}{a} \log \left(\frac{1+a h \sigma_{0}}{1+a \widetilde{x}}\right),
$$

we obtain a system of two equations (for $\widetilde{x}$ and $\sigma_{0}$ ) for determining the point $\sigma=-\sigma_{0}, x=-h \sigma_{0}$ on the limit cycle of the comparison system. In this case, we have a system of two equations, which is much simpler than $(3.5)$.

Note that the limit cycle of the original system can be fairly accurately estimated by applying comparison systems (see Fig. 4).

\section{CONCLUSIONS}

We considered the anti-windup flowchart with an unstable object (i.e. with a first-order link), which has become standard over the last decade. Keldyshs method was developed for stability domain analysis. The results suggest that the stability domain tends to infinity as the parameter a approaches zero. 

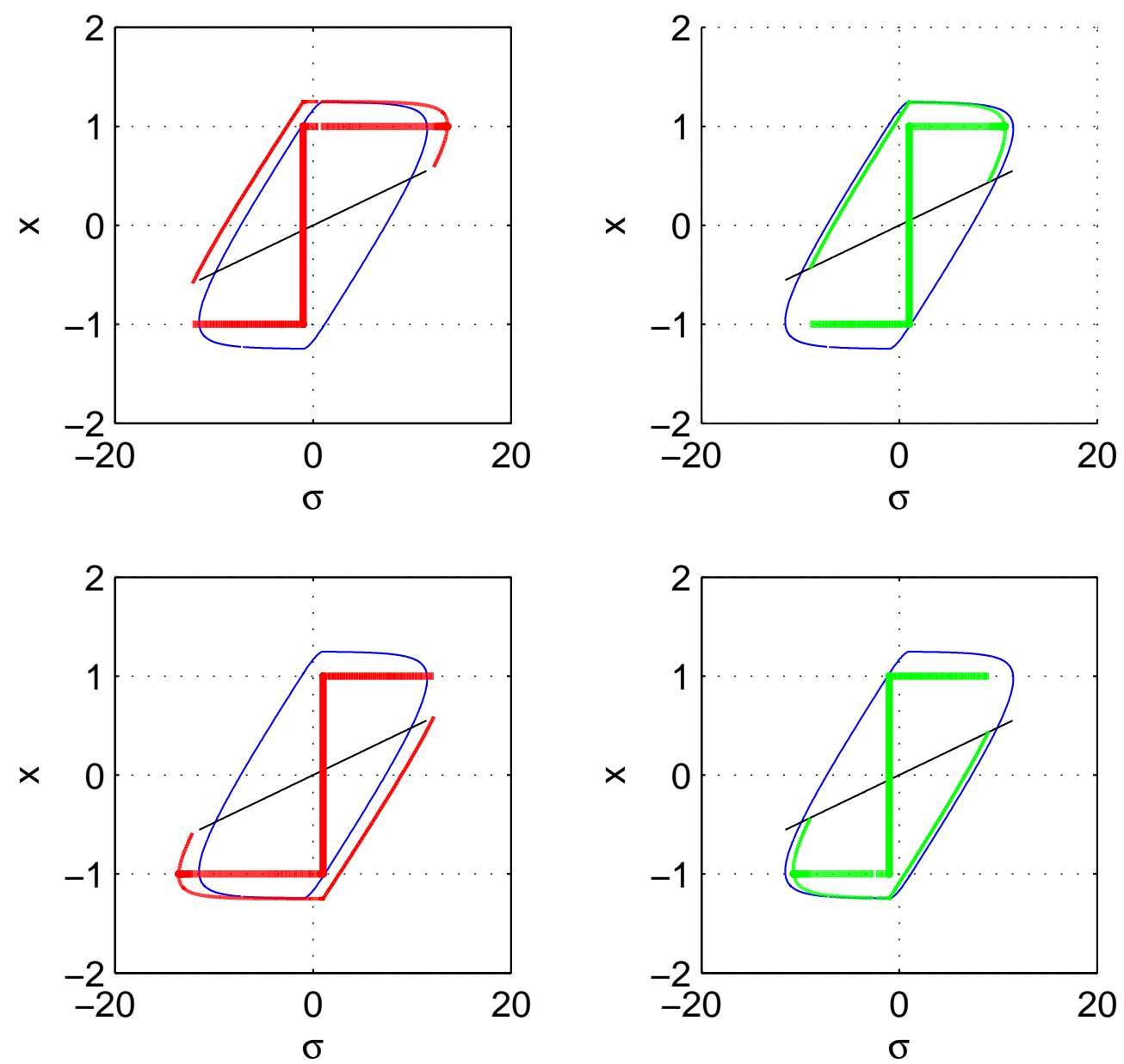

Figure 3: Limit cycle of the original system and the construction of limit cycles in comparison systems with the switching curve for nonlinearities taken into account. 


\section{References}

[1] Kuznetsov, N. V., Leonov, G. A. \& Seledzhi, S. M. [2011] Hidden oscillations in nonlinear control systems, IFAC Proceedings Volumes (IFAC-PapersOnline) 18, 2506-2510, doi:10.3182/20110828-6-IT-1002.03316.

[2] Leonov, G. \& Kuznetsov, N. V. [2013] Analytical-numerical methods for hidden attractors' localization: the 16th Hilbert problem, Aizerman and Kalman conjectures, and Chua circuits, Numerical Methods for Differential Equations, Optimization, and Technological Problems, Computational Methods in Applied Sciences, Volume 27, Part 1 (Springer), pp. 41-64, doi:10.1007/978-94-007-5288-7_3.

[3] Leonov, G. A., Bragin, V. O. \& Kuznetsov, N. V. [2010] Algorithm for constructing counterexamples to the Kalman problem, Doklady Mathematics 82, 540-542, doi:10.1134/S1064562410040101.

[4] Leonov, G. A., Bragin, V. O. \& Kuznetsov, N. V. [2010] On problems of Aizerman and Kalman, Vestnik St. Petersburg University. Mathematics 43, 148-162, doi:10.3103/S1063454110030052.

[5] Leonov, G. A. \& Kuznetsov, N. V. [2011] Algorithms for searching hidden oscillations in the Aizerman and Kalman problems, Doklady Mathematics 84, 475-481, doi:10.1134/S1064562411040120.

[6] Leonov, G. A. \& Kuznetsov, N. V. [2011] Analytical-numerical methods for investigation of hidden oscillations in nonlinear control systems, IFAC Proceedings Volumes (IFAC-PapersOnline) 18, 24942505, doi:10.3182/20110828-6-IT-1002.03315.

[7] Bragin, V. O., Vagaitsev, V. I., Kuznetsov, N. V. \& Leonov, G. A. [2011] Algorithms for finding hidden oscillations in nonlinear systems. The Aizerman and Kalman conjectures and Chua's circuits, Journal of Computer and Systems Sciences International 50, 511-543, doi:10.1134/S106423071104006X.

[8] Lauvdal, T., Murray, R. \& Fossen, T. [1997] Stabilization of integrator chains in the presence of magnitude and rate saturations: a gain scheduling approach, Proc. IEEE Control and Decision Conference, pp. 4404-4005.

[9] National Research Council. Aviation Safety and Pilot Control (Washington, D.C.: National Academy Press, 1997)

[10] M.A. Dornheim. Report pinpoints factors leading to yf-22 crash. Aviation Week and Space Technology, $137: 53-54,1992$

[11] C.A. Shifrin. Sweden seeks cause of gripen crash. Aviation Week and Space Technology, 139:78-79, 1993.

[12] L. Zaccarian, A. Teel, Modern Anti-windup Synthesis: Control Augmentation for Actuator Saturation, Princeton University Press, Princeton Series in Applied Mathematics, 2011.

[13] A. Saberi, Z. Lin, and A.R. Teel. Control of linear systems with saturating actuators. IEEE Trans. on Automatic Control, 41(3):368378, 1996.

[14] N. Kapoor, A. R. Teel, and P. Daoutidis. An anti-windup design for linear systems with input saturation. Automatica, 34(5):559574, 1998.

[15] S. Galeani, M. Massimetti, A. R. Teel, and L. Zaccarian. Reduced order linear anti-windup augmentation for stable linear systems. International Journal of Systems Science, 37:115127, 2006.

[16] G. Grimm, J. Hatfield, I. Postlethwaite, A.R. Teel, M.C. Turner, and L. Zaccarian. Anti-windup for stable linear systems with input saturation: an LMI based synthesis. IEEE Transactions on Automatic Control, 48(9):15091525, 2003. 
[17] S. Tarbouriech, M. Turner, Anti-windup design: an overview of some recent advances and open problems, IET Control Theory and Applications, 2009, 3 (1), pp. 1-19.

[18] M. V. Keldysh, Selected Works: Mechanics (Nauka, Moscow, 1985) [in Russian].

[19] A. A. Andronov, A. A. Vitt, and S. E. Khaikin, Theory of Oscillations (ONTI, Moscow, 1937; Pergamon, London, 1966).

[20] G. A. Leonov, Autom. Remote Control 66, 10591068 (2005).

[21] G. A. Leonov, Vestn. Sankt-Peterburg. Gos. Univ., Ser. 1: Mat. Mekh. Astron., No. 4, 4878 (2006). 